\title{
Surface and interstitial Ti diffusion at the rutile TiO2(110) surface
}

Article

Accepted Version

Accepted version using RSC template- please check copyright etc.

Mulheran, P. A., Nolan, M., Browne, C. S., Basham, M., Sanville, E. and Bennett, R. A. (2010) Surface and interstitial Ti diffusion at the rutile TiO2(110) surface. Physical Chemistry Chemical Physics, 12 (33). pp. 9763-9771. ISSN 1463-9076 doi: https://doi.org/10.1039/C002698K Available at https://centaur.reading.ac.uk/5776/

It is advisable to refer to the publisher's version if you intend to cite from the work. See Guidance on citing.

To link to this article DOI: http://dx.doi.org/10.1039/C002698K

Publisher: Royal Society of Chemistry

All outputs in CentAUR are protected by Intellectual Property Rights law, including copyright law. Copyright and IPR is retained by the creators or other copyright holders. Terms and conditions for use of this material are defined in the End User Agreement.

\section{www.reading.ac.uk/centaur}

\section{CentAUR}

Central Archive at the University of Reading 
Reading's research outputs online 


\title{
Surface and Interstitial Ti Diffusion at the Rutile $\mathrm{TiO}_{2}(110)$ Surface
}

\author{
P.A. Mulheran, ${ }^{* a}$ M. Nolan ${ }^{* b}$, C.S. Browne ${ }^{c}$, M. Basham ${ }^{d}$, E. Sanville ${ }^{e}$ and R.A. Bennett ${ }^{f}$ \\ Received (in $X X X, X X X) X_{\text {th }} X X X X X X X X X 200 X$, Accepted Xth $X X X X X X X X X 200 X$ \\ First published on the web Xth $X X X X X X X X X 200 X$ \\ ${ }_{s}$ DOI: 10.1039/b000000x
}

Diffusion of Ti through the $\mathrm{TiO}_{2}(110)$ rutile surface plays a key role in the growth and reactivity of $\mathrm{TiO}_{2}$. To understand the fundamental aspects of this important process, we present an analysis of the diffusion of $\mathrm{Ti}$ adspecies at the stoichiometric $\mathrm{TiO}_{2}(110)$ surface using complementary computational methodologies of density functional theory corrected for on-site Coulomb

10 interactions $(\mathrm{DFT}+\mathrm{U})$ and a charge equilibration $(\mathrm{QEq})$ atomistic potential to identify minimum energy pathways. We find that diffusion of Ti from the surface to subsurface (and vice versa) follows an intersticialcy exchange mechanism, involving exchange of surface Ti with the 6-fold coordinated Ti below the bridging oxygen rows. Diffusion in the subsurface between layers also follows an interstitialcy mechanism. The diffusion of $\mathrm{Ti}$ is discussed in light of continued attempts 15 to understand the re-oxidation of non-stoichiometric $\mathrm{TiO}_{2}(110)$ surfaces.

\section{Introduction}

Titanium dioxide is a technologically important material and has garnered considerable attention as a possible source of clean energy by photocatalytic water splitting, as a cleanup 20 technology in waste streams and when combined with suitable supported metal catalysts as an environmental gas purifier. Titania also has applications in coatings and sensors ${ }^{1}$. Besides these applications, it is one of the most widely studied prototypical reducible metal oxides with the Ti having many 25 stable oxidation states producing a complex structural phase diagram ${ }^{2}$. Stoichiometric $\mathrm{TiO}_{2}$ has four polymorphs: rutile, anatase, brookite and cotunnite ${ }^{3}$. Nearly stoichiometric rutile shows two homologous series of planar bulk defects that selfassemble into crystallographic shear planes: $\mathrm{Ti}_{\mathrm{n}} \mathrm{O}_{2 \mathrm{n}-1}$ with $30(4<\mathrm{n}<10)$ based on $\{121\}$ directed planes; and $(16<\mathrm{n}<\sim 37)$ based on $\{132\}^{4-6}$. Stable reduced phases down to $\mathrm{Ti}_{4} \mathrm{O}_{7}$, a crystal structure with promising electrical characteristics ${ }^{7}$, can also be formed. $\mathrm{Ti}_{2} \mathrm{O}_{3}$ is a corundum structured pure $\mathrm{Ti}^{3+}$ phase which also appears as a reduced surface phase on near 35 stoichiometric rutile surfaces treated in vacuum ${ }^{8}$. The equilibrium phases make an interesting system in which to modify the electrical and structural characteristics of the oxide. The atomistic processes that allow the system to move, for example, from stoichiometric rutile to a self-assembled 40 planar array of defects in the bulk upon reduction, are somewhat hidden from view. However, recent experimental work has highlighted some key constituents to the puzzle, notably by considering the reverse process, that is the reoxidation of non-stoichiometric material $^{9-11}$.

${ }_{45}$ The rutile (110) surface has been extensively investigated experimentally over a number of years by surface science techniques, and usually on electrically conducting bulk reduced crystals that have changed colour which indicates a non-stoichiometry. Early work on re-oxidation by Henderson 50 suggested $\mathrm{Ti}$ interstitials were the key point defect in nonstoichiometric rutile ${ }^{12}$. Indeed Scanning Tunnelling Microscopy (STM) studies by Onishi and Iwasawa showed how these defects can move from bulk to surface to grow new surface phases, the so-called added row $\mathrm{Ti}_{2} \mathrm{O}_{3}$ reconstruction ${ }^{8}$. ${ }_{55}$ At elevated temperature, however, variable temperature STM revealed how the (110) surface of a reduced crystal re-grows new layers of $\mathrm{TiO}_{2}$ (in contrast to $\mathrm{Ti}_{2} \mathrm{O}_{3}$ ) in a rather complex layer-by-layer fashion when exposed to oxygen ${ }^{9,10}$. These studies show that the reduced crystal contains a solid solution 60 of $\mathrm{Ti}$ interstitials that are mobile above $\sim 473 \mathrm{~K}$ and able to diffuse to the surface to react with the impinging oxygen (or other adsorbed species such as formate ${ }^{13}$ ). The relationship between the reduction and re-oxidation steps has been demonstrated by further experiments on the self-doping of 65 titania crystals and ultra-thin films which show how deposited $\mathrm{Ti}$ adatoms can diffuse into the bulk as interstitials when annealed $^{14}$. Ti interstitials and adatoms are therefore of paramount importance to the surface structures, ${ }^{15}$ the surface chemistry $^{16}$ and the growth of titania, and it is desirable to 70 have modelling schemes that can describe these species, their reactivity and their mobility ${ }^{17}$. Modelling schemes that accurately represent electronic and geometric structures are particularly challenging for non-stoichiometric materials with variable oxidation states.

75 The description of reduced rutile has been an active topic for many years, with much discussion as to whether the dominant species involved are Ti interstitials ${ }^{15}$ or oxygen vacancies ${ }^{18}$. Both defects can produce the same signature in ultra violet photoemission spectroscopy and in density functional theory 80 (DFT) simulations ${ }^{19}$, i.e. reduced $\mathrm{Ti}^{3+}$ ions. However, this debate is not the reason we study Ti interstitials. Rather, given that these species are key to the growth of $\mathrm{TiO}_{2}$ through their diffusion from bulk to the surface and reaction with oxygen, we are studying the diffusion of Ti interstitials as a first step 85 to providing a deeper understanding of this process.

Reference 17 provides a thorough exploration of the diffusion of $\mathrm{Ti}$ and $\mathrm{O}$ ad-species on the rutile (110) surface using density functional theory (DFT) with the local density approximation (LDA). However, it is known that this 90 approach will not describe well the localisation of d-electrons that are expected in reduced titania. Therefore we have 
undertaken a series of calculations using DFT $+\mathrm{U}$ to correct for on-site Coulomb interactions, providing a good comparator for the LDA results and allowing confrontation with experiment to assess the relative merits of the approaches.

5 In particular, in recent work we have presented direct experimental valdiation of the $\mathrm{DFT}+\mathrm{U}$ computational approach, applied in this paper. We studied self-doped titania films, characterising the electronic properties of surface adsorbed $\mathrm{Ti}^{20}$. We found that an approach such as DFT+U is 10 indeed needed to interpret the experimental results and consistently describe the $\mathrm{Ti}^{3+}$ ions present in this system. The best agreement with the spectroscopy of the gap states induced by the adsorbed adatom is obtained with $\mathrm{U}=3 \mathrm{eV}$. This result is consistent with the value of $U$ assigned in 15 calculations of oxygen vacancies in the (110) surface ${ }^{21}$, which create a reduced surface Ti defect state in the band gap. These latter calculations share important similarities with B3LYP results $^{22}$ which also localise the Ti $3 \mathrm{~d}$ states.

In this paper we employ experimentally benchmarked 20 calculations of static structures ${ }^{20}$ to consider the dynamical aspects of $\mathrm{Ti}$ adatom and interstitial mobility in the rutile (110) surface. We find key barriers and transition pathways in the surface and subsurface regions and explore their electronic structure. The results are contrasted with those of reference

2517 , and are found to be in much better agreement with experimental results. Furthermore, we believe the charge localisation we predict facilitates new physical insight into surface growth and reactivity.

In our work, the DFT+U methodology is used to investigate 30 the stability and properties of the $\mathrm{Ti}$ interstitials, since it is known to provide a consistent description of reduced $\mathrm{Ti}^{20,21}$. To investigate the diffusion pathways between the various adatom and interstitial sites, of which there are many possibilities, we first employ atomistic calculations using the ${ }_{35}$ charge equilibration (QEq) methodology ${ }^{17,}{ }^{23-31}$ In order to provide a realistic set of minimum energy pathways (MEP) for full investigation with $\mathrm{DFT}+\mathrm{U}$, we consider the effectiveness of the QEq approach in describing the energy landscape of the defects, and find that some modification to 40 the original model is required. The modification employed involves altering the degree of charge transfer between species in the model, in particular restricting the transfer to $\mathrm{Ti}$ species only. This change is grounded in our analysis of the $\mathrm{DFT}+\mathrm{U}$ calculations, and is consistent with experimental 45 observations. With the modified potential shown to describe interstitial $\mathrm{Ti}$ consistently, we then screen a number of diffusion pathways, and select the most energetically favourable for further quantification using constrained minimisation in DFT+U calculations. The QEq approach has 50 the appealing quality of being able to describe defects such as

$\mathrm{Ti}$ interstitials and their diffusion and is an appropriate method to use for modelling of the dynamics of Ti diffusion and reaction with oxygen to ultimately study the dynamics of $\mathrm{TiO}_{2}$ growth.

${ }_{55}$ The layout of the paper is as follows. In the following section, we describe the computational methodologies. In section 3 we present results, first for the structures and energies of the interstitials and adatoms using the DFT $+\mathrm{U}$ and atomistic QEq calculations, and describe the impact of our charge transfer 60 modification on the latter. In Section 3.2 we present results for our Minimum Energy Pathways for diffusion in the selvedge. Discussions of our results, focussing on charge transfer during diffusion, follow in Section 4 along with conclusions for the implications of our results for ${ }_{65}$ experimental interpretation and future dynamical simulation of surface growth.

\section{Methodology}

\subsection{DFT+U Calculations}

All our first principles calculations are carried out in the 70 framework of periodic plane wave density functional theory $(\mathrm{DFT})^{32}$. In this approach, the valence electronic states are expanded in a basis of periodic plane waves, with an energy cut-off of $396 \mathrm{eV}$, while the core-valence interaction is treated using PAW potentials ${ }^{33}$. The PW91 exchange-correlation 75 functional is used throughout. The surface is modelled as a finite thickness slab model with three-dimensional periodicity. Surface slabs along the $\mathrm{z}$-direction (perpendicular to the surface) are separated using a vacuum thickness of $\sim 10$ '. For the interstitial calculations, a single $\mathrm{Ti}$ is placed in two 80 different sites in the first, second and third (bulk-like) subsurface O-Ti-O layers. Full relaxation is performed, except for the bottom O-Ti-O unit. We refer to our previous work for details of the $\mathrm{Ti}$ adatoms adsorbed at the rutile (110) surface $^{20}$.

${ }_{85}$ The slab model is 6 O-Ti-O units deep with a $(2 \times 4)$ surface cell expansion, minimising periodic defect-defect interactions, and providing a concentration of adsorbed $\mathrm{Ti}$ atoms that matches the coverage used in self-doping experiments ${ }^{20}$. kpoint sampling is at the $\Gamma$-point and the Methfessel-Paxton 90 smearing scheme with a smearing parameter of $0.1 \mathrm{eV}$ is used. As discussed in the introduction, an important aspect of these calculations concerns the theoretical description of the reduced Ti ions. Reduced Ti ions have partially occupied $3 d$ shells, which can be difficult to describe with approximate ${ }_{5}$ DFT exchange-correlation functionals ${ }^{21,21,34-37}$. It has already been demonstrated that the DFT+U approach ${ }^{38,39}$ can be successfully applied to study non-stiochiometric $\mathrm{TiO}_{2}{ }^{20,21,34-}$ ${ }^{37}$ and we continue to use DFT $+\mathrm{U}$ in this work. The formalism due to Dudarev et al. ${ }^{39}$ as implemented in VASP is employed. 100 The quantity $(\mathrm{U}-\mathrm{J}$ ) is used, where $\mathrm{J}$ is the exchange parameter. However, since the system is not magnetic, we set $\mathrm{J}$ to be zero, so that $(\mathrm{U}-\mathrm{J}) \equiv \mathrm{U}$. From our previous work ${ }^{20}, \mathrm{U}$ is set to $3 \mathrm{eV}$.

The charge density from the converged VASP calculations 105 was partitioned to the ions using our own Bader analysis code $^{40}$, allowing comparisons to be made to the charge distributions predicted through the QEq approach described next.

\section{${ }_{110}$ 2.2 QEq Calculations}

The DFT $+\mathrm{U}$ methodology leads to stronger localisation of charges in non-stoichiometric or defective structures in comparison to standard $\mathrm{DFT}^{20,21}$. This physical picture 
strengthens the possibility of using empirical potential schemes which employ charges fixed spatially to ion cores in the lattice and especially those models which have the flexibility to allow charges to move. One such leading scheme 5 is the $\mathrm{QEq}$ methodology ${ }^{23}$ which allows charge transfer between ions to minimise the electrostatic energy $\mathrm{E}_{\mathrm{es}}$, whilst including contributions from the charging energies for each ion which takes a parabolic form:

$E_{g g}=\sum_{i}\left(E_{i}^{0}+\chi_{i} q_{i}+l_{i}^{0} p_{i}^{2}\right)+\sum_{i} \sum_{i} p_{i} p_{i}(1)$

10 Here $\chi_{i}^{0}$ and $J_{i}^{0}$ are related to the ions' electronegativity and hardness. $J_{i j}$ is the shielded Coulomb interaction between ions, the shielding being estimated by the overlap of s-type Slater orbitals. For any given ionic configuration, the charge $q_{i}$ on the ions is adjusted to minimise eqn. (1) under the constraint 15 of conserved total charge $\sum_{i} p_{i}=\mathbb{q}$, without moving the ionic positions. Once this equilibration has been achieved, the resultant forces on the ions can be used to move them as in usual minimisation or dynamics procedures ${ }^{25}$. The atomistic model for the material is completed by the addition of short20 range potentials to represent the covalent bonding which is known to be significant for titania ${ }^{1}$. Hallil et al have developed a suitable model for titania, using a pair-functional form to describe Ti-O covalent bonds ${ }^{28}$.

In our QEq calculations we use the same rutile surface slabs ${ }_{25}$ described above for the $\mathrm{DFT}+\mathrm{U}$ calculations. The lattice parameters were fixed to bulk values, and the ion coordinates relaxed. For both $\mathrm{DFT}+\mathrm{U}$ and the $\mathrm{QEq}$ calculations, results with larger cells, larger vacuum gaps, and with two adsorbed adatoms either side of the slab (for the $\mathrm{Ti}$ adatom 30 calculations) produce essentially identical results; more details are in ref. 20. The geometry minimisations were performed using a conjugate gradient method and we use our own in-house code to perform all the QEq calculations.

Minimum Energy Pathways for the interstitial and adatom 35 diffusion are created with the QEq atomistic potential using the nudged elastic band method ${ }^{41}$. Selected pathways are further investigated using constrained minimisation with $\mathrm{DFT}+\mathrm{U}$. Here the position of the migrating species is held fixed whilst the surrounding atoms are relaxed. This 40 procedure is performed for each image along the pathway, thereby assessing the pathway's viability and obtaining another estimate of the activation energy.

\section{Results}

\subsection{The structure and energy of interstitials in the (110) 45 surface}

In reference 20 we presented results for the $\mathrm{Ti}$ adatom at the rutile (110) surface. Two stable binding sites are possible, the more favourable of which positions the adatom between two bridging and one in-plane oxygen. Due to the symmetry, we
50 referred to it as the ih $\operatorname{site}^{20}$. In this paper, we find it convenient to change our notation where this adsorption site will henceforth be labelled A; see Figure 1. An alternative stable adsorption site is possible where the adatom is positioned between 2 in- plane and one bridging oxygen, 55 labelled site B in Figure 1 and throughout the paper (it was labelled $i v$ in ref. 20).

Within our DFT $+\mathrm{U}$ methodology, we calculated that site A is $0.34 \mathrm{eV}$ lower in energy than site $\mathrm{B}$, however, a barrier for diffusion between $\mathrm{B}$ to $\mathrm{A}$ is present since $\mathrm{B}$ is found to be 60 stable upon relaxation.

An important aspect of the DFT $+\mathrm{U}$ approach is to relate the localisation of charge to the defect-induced gap state observed in experiment. A convenient representation is found by plotting the spin iso-surfaces for the four extra unpaired d${ }_{65}$ electrons present in the surface with adsorbed Ti. Following this scheme, we present in Figure 2 the spin iso-surfaces with interstitials in the first, second and third O-Ti-O layers beneath the (110) surface. These sites are all have the same symmetry as the adatom in site A. In Figure 1 these sites are 70 indicated as Fi in the first layer, and as shown they tend to sit beneath in-plane oxygen in plan view rather than directly beneath the site A adatom. The adatoms in the corresponding site in layers 2 and 3 are referred to as Fii and Fiii in the following section. The spin density plots in figure 2 show 75 reduced $\mathrm{Ti}$ ions present - in particular the interstitial Ti has a $\mathrm{Ti}^{3+}$ oxidation state and a small number of neighbouring $\mathrm{Ti}$ atoms are also reduced to $\mathrm{Ti}^{3+}$. Note that the electrons introduced by $\mathrm{Ti}$ are predominantly localised. In previous work, using hybrid $\mathrm{DFT}^{42}$, Finazzi et al. also showed the 80 formation of localised $\mathrm{Ti}^{3+}$ ions when a $\mathrm{Ti}$ interstitial is present, giving good confidence in our use of DFT $+\mathrm{U}$.

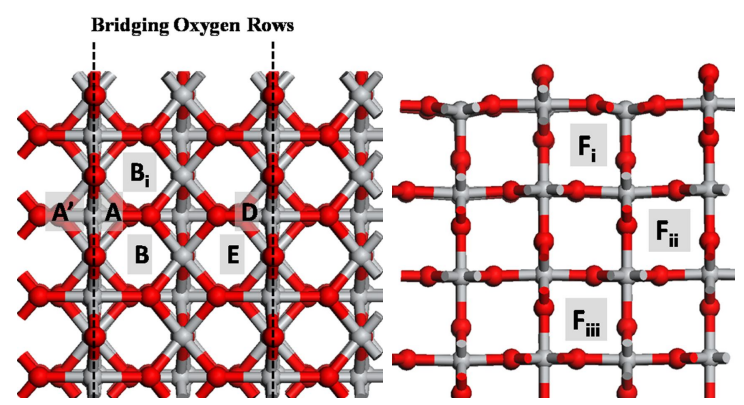

Fig.1 Views of the (110) rutile surface indicating the adatom and 85 interstitial sites used in this paper. Ti atoms are grey and $O$ atoms are red. The Bridging oxygen rows are indicated with the dashed lines. Site $A$ (and equivalents $A$ ' and $D$ ) is the adatom in its most favourable site, next to two bridging oxygen and one in-plane oxygen. Site $B$ (and its equivalent $E$ ) is the adatom in its second favourable binding site next to 90 one bridging and 2 in-plane oxygen. Site Fx is an interstitial site $x O-T i-O$ layers down with the same symmetry as site A; there is an alternative interstitial site $B x$, with the same symmetry as site $B$. 

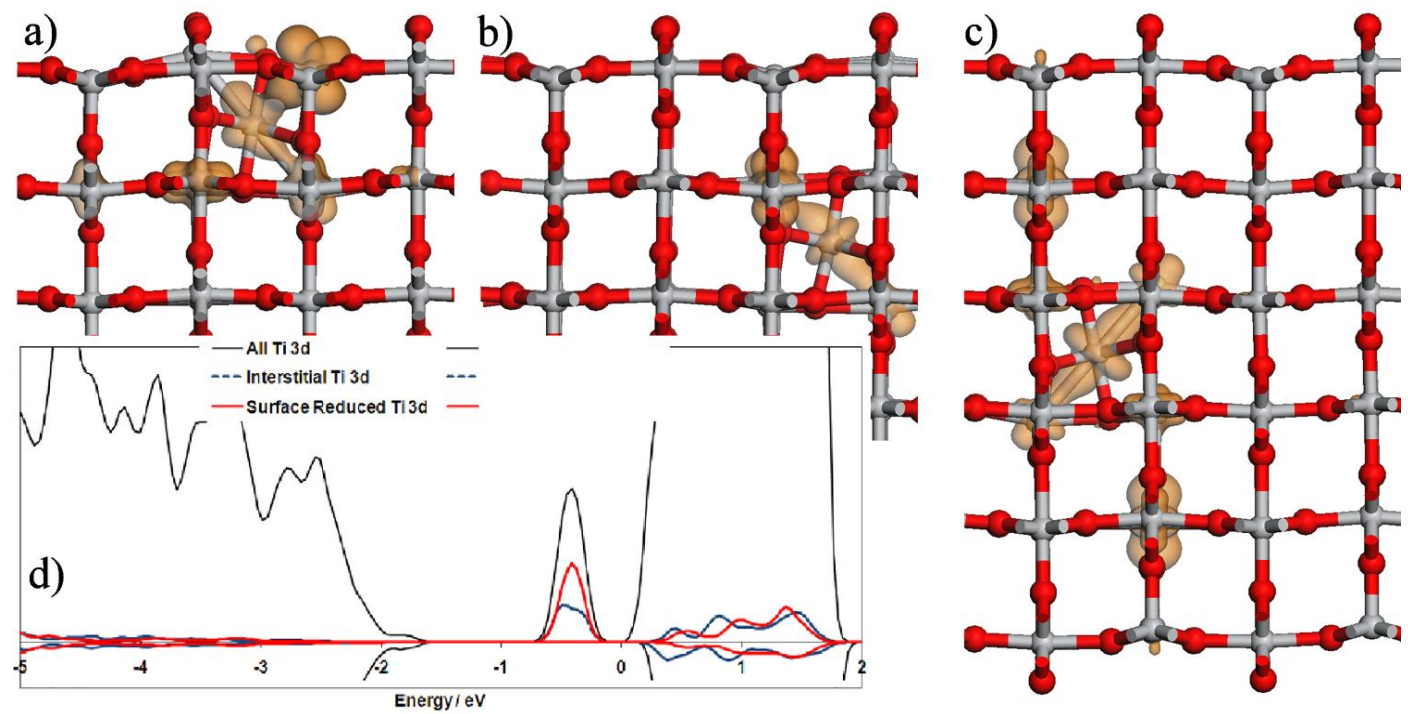

Fig2 The spin density plots from the DFT+U interstitials (a) Fi, (b) Fii and (c) Fiii. Also shown in (d) is the projected electronic density of states, showing the Ti $3 d$ derived gap state induced by the Fi interstitial

Also shown in Figure 2 is the projected electron density of ${ }_{5}$ states for the Fi interstitial site. This shows the defect-induced gap state associated with $\mathrm{Ti}^{3+}$, arising from the interstitial atoms and reduction of neighbouring lattice Ti. This contrasts to the split level found for the adatom in site $\mathrm{A}$, where $\mathrm{T} \mathrm{Ti}^{2+}$ signature is observed for the adatom, and a $\mathrm{Ti}^{3+}$ feature for an 10 in-plane five-fold coordinated $\mathrm{Ti}^{3+}$. In both cases, the remaining electron is spread across other lattice Ti. The corresponding results for the interstitial in the $\mathrm{Bi}$ site are not shown here but are broadly similar. They show surface 5 -fold Ti reduced to $3+$, the interstitial maintaining a $3+$ oxidation is state and the remaining charge spread over a small number of subsurface Ti.

We have also calculated the relaxed structure and energies of the interstitials using the QEq methodology ${ }^{31}$. In Figure 3 we show the relative energetics for the Ti adatom and interstitials 20 from DFT $+\mathrm{U}$ and QEq calculations. With the energy of the A site adatom set to $0 \mathrm{eV}$ for $\mathrm{DFT}+\mathrm{U}$ and $\mathrm{QEq}$, the figure shows the relative energetics of the $\mathrm{B}$ site adatom and the corresponding interstitial sites. Both $\mathrm{DFT}+\mathrm{U}$ and $\mathrm{QEq}$ find the same stable sites, and furthermore the binding to the site A is 25 the most favourable adatom site in both schemes, with an energy difference between $\mathrm{A}$ and $\mathrm{B}$ sites of $0.56 \mathrm{eV}$ in the $\mathrm{QEq}$ scheme respectively (c.f $0.34 \mathrm{eV}$ in $\mathrm{DFT}+\mathrm{U})$.

The difference in binding energy between the interstitial $\mathrm{Fi}$ and $\mathrm{Bi}$ sites $(0.09 \mathrm{eV}$ and $0.07 \mathrm{eV}$ respectively) is much less 30 marked than that between A and B in both the DFT+U and the QEq calculations, as might be anticipated since in bulk these sites would be identical by symmetry.

However, there is a marked difference between the energy of the adatom $(\mathrm{A}, \mathrm{B})$ and interstitial $(\mathrm{Fi}, \mathrm{Bi})$ sites calculated by ${ }_{35} \mathrm{DFT}+\mathrm{U}$ and the Hallil QEq model. The DFT $+\mathrm{U}$ calculation shows the interstitials are more energetically favourable than adatoms by $0.6 \mathrm{eV}$ and $0.8 \mathrm{eV}$ respectively for the $\mathrm{A}$ and $\mathrm{B}$ sites, whereas the QEq differences are very much larger at $2.5 \mathrm{eV}$ and $3.0 \mathrm{eV}$. This is a significant difference in the energy 40 landscapes of the models. Whilst our finding that $\mathrm{Ti}$ interstitials are more energetically favourable than adatoms agrees with experimental results for adatoms diffusing down into the bulk upon annealing ${ }^{14}$, trapping them in the bulk by such a large amount as $2.5 \mathrm{eV}$ does not accord with the re45 growth of reduced rutile at elevated temperature, even if oxygen ad-species promote the growth ${ }^{9,10,15}$. We conclude that the empirical QEq potential of Hallil et $a l^{28}$ overestimates the adsorption energy of the interstitials with respect to the adatom energy.

50

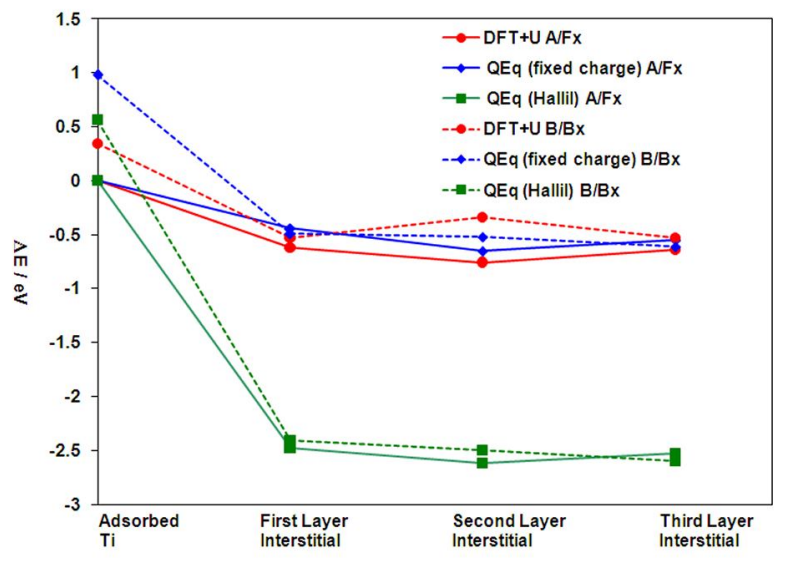

Fig.3 Relative energies of different Ti sites from the DFT $U$ and $Q E q$ calculations. The energy of the Ti adatom in the A site is set to zero

${ }_{55}$ The reason for the discrepancy in the adatom-interstitial binding energy differences can be traced to the charge transfer occurring in the QEq potential at the (110) surface. In Figure 4 we show the $\mathrm{QEq}$ charges on the ions in the relaxed structures with the adatom in the surface $\mathrm{A}$ and $\mathrm{B}$ sites and the ${ }_{60}$ first layer instersitial Fi and Bi sites, as well as the labelling for Tables 1 and 2. In this figure, the radius of the ions reflects their excess charge (in $\mathrm{e}^{-}$) over bulk values in order to provide a pictorial view of the charge distribution. For 
comparison, Bader analysis is used to partition the charge to the ions in the DFT $+\mathrm{U}$ results. The charges on the ions are given in Tables 1 and 2, and for clarity we also provide the pictorial view of the charge distributions for all calculations in 5 the Supplementary Information.

Referring to Tables 1 and 2, it is clear that there is a qualitative difference between the behaviour of the Ti and the $\mathrm{O}$ atoms in the two approaches. In the case of the Ti, the QEq charges are broadly correlated with the Bader charges. In 10 contrast, the distribution of charges on the $\mathrm{O}$ atoms do not correlate well between the calculations. In particular, the Bader analysis of the DFT $+U$ reveals only small excess charges on the $\mathrm{O}$ atoms, within the limits of about $+/-0.1 \mathrm{e}^{-}$, whereas the QEq model gives a much wider range of excess $\mathrm{O}$ 15 charges in the range of about $+/-0.4 \mathrm{e}^{-}$.

This charging of the oxygen nearest-neighbours by the $\mathrm{Ti}$ interstitial in the QEq model causes a favourable decrease in electrostatic energy with small penalties in self energy (see equation 1) and from the short range potential. These combine 20 to lead to an elastic distortion in the surrounding lattice with ions moving closer together, screening the interstitials and significantly lowering the total energy. The response of the $\mathrm{Ti}$ adatom does not show such a strong effect since the adatom has fewer oxygen neighbours to charge.

${ }_{25}$ The behaviour of the oxygen within the Hallil model suggests that the energetics of the QEq component underplay the costs of moving the charge both to and from the oxygen. To understand the consequences of this, we have repeated our calculations keeping the charge on all of the oxygens fixed to 30 their bulk value of $1.26 \mathrm{e}^{-}$, with only the $\mathrm{Ti}$ ions able to transfer charge between themselves using the original charging self-energies ${ }^{28}$. We show in Figure 3 how the energy landscape of the defects, measured relative to the energy of the adatom in site $\mathrm{A}$ in each model, is now in much better 35 agreement with the $\mathrm{DFT}+\mathrm{U}$ results.

Table 1 The excess charge dQ, measured in units of e $\mathrm{e}^{-}$, over bulk values for the ions labelled in Figs. 4a and 4b using the Hallil QEq potential, $\mathrm{DFT}+\mathrm{U}$ and fixed $\mathrm{O}$ - charge $\mathrm{QEq}$.

\begin{tabular}{cccc} 
A adatom & Hallil QEq & DFT + U & Fixed O QEq \\
\hline & & & \\
\hline T1 & 0.775 & 0.585 & 0.825 \\
T2 & 0.215 & 0.074 & 0.348 \\
T3 & 0.010 & -0.007 & -0.056 \\
O1 & 0.370 & 0.127 & 0.0 \\
O2 & 0.047 & 0.000 & 0.0
\end{tabular}

\begin{tabular}{cccc} 
B adatom & Hallil QEq & DFT $+\mathrm{U}$ & Fixed O QEq \\
\hline T1 & 0.812 & 0.990 & 0.825 \\
T2 & 0.109 & 0.399 & 0.130 \\
T3 & 0.273 & 0.006 & 0.420 \\
O1 & 0.343 & 0.139 & 0.0 \\
O2 & 0.011 & 0.016 & 0.0 \\
O3 & -0.355 & -0.073 & 0.0
\end{tabular}

40
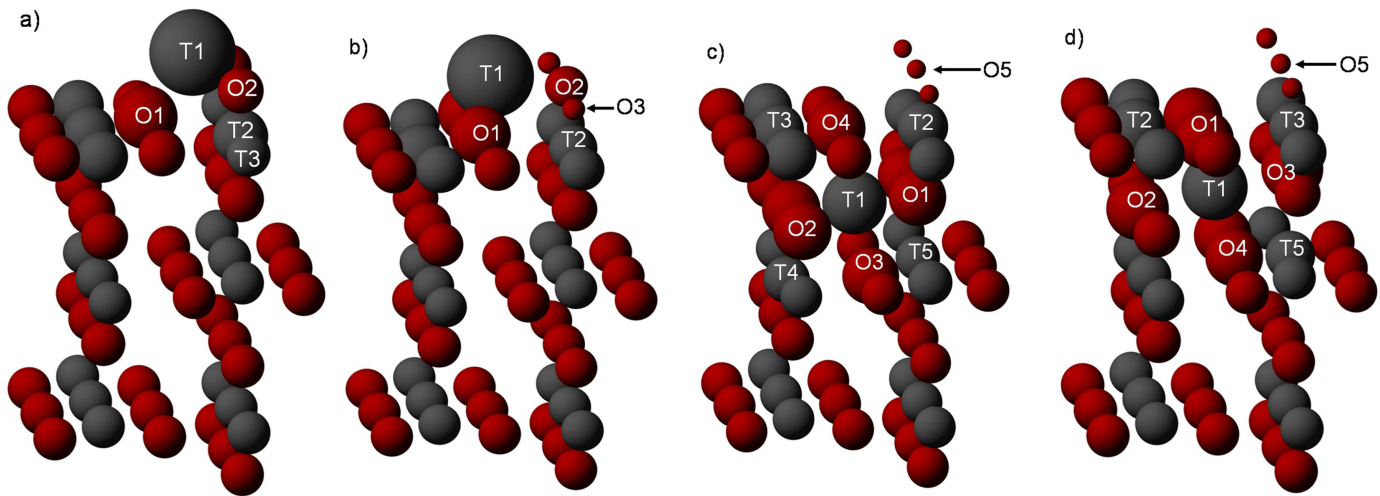

Fig. 4 (a) Hallil model QEq results for the A adatom site; (b) Hallil model QEq results for the B site; (c) Hallil model QEq results for the Fi site and (d) Hallil model QEq results for the Bi site. The oxygen are red and the titanium dark grey. The size of the ions in all images represent how much excess charge $d Q\left(e^{-}\right)$is associated with the ion in comparison to its bulk charge (specifically 45 radius scales as $1+1.3 d Q$.). The values of $d Q$ for the labelled ions are given in Tables 1 and 2 . Only a section of the full cell used in the calculations is show for clarity, with the upper surface being the free one with the exposed bridging oxygen.

50 In support of the physical basis of this modification to the QEq model, experimental data for the core-level shifts in $\mathrm{x}$ ray photoelectron spectroscopy of stoichiometric $\mathrm{TiO}_{2}(110)$, $\mathrm{Ti}$ adatom doped $\mathrm{TiO}_{2}(110)$, and added row $\mathrm{Ti}_{2} \mathrm{O}_{3}$ reconstructed $\mathrm{TiO}_{2}(110)$ are presented in the Supporting

55 Information. All cases show very similar O1s lineshapes with no spectral features attributable to large variances in oxygen charge state. This confirms that the charge transfer to and from the oxygen atoms of the $\mathrm{TiO}_{2}(110)$ surface upon $\mathrm{Ti}$ adsorption predicted by our calculations should be minimal.

\section{3.2 Minimum Energy Pathways for Ti interstitial Diffusion}

Detailed results for NEB calculations of Ti diffusion with the QEq potentials have previously been presented in reference 31 , to which we refer for further details. A summary of the activation energies of feasible diffusion pathways is given in ${ }_{65}$ Table 3, obtained with the modified (fixed oxygen charge) QEq model. From these, we select the most favourable pathways for further investigation with DFT+U.

The first pathway we consider for the diffusion between adatom and first layer interstitial is A-Fi via exchange with a 
6-fold coordinated lattice Ti underneath the bridging oxygen row. In Figure 5 we show the energies of the images along the relaxed NEB pathway, using the starting site A adatom QEq energy as a convenient zero of energy. For alternate images 5 along the path, we apply the constrained minimisation in $\mathrm{DFT}+\mathrm{U}$ and plot the relaxed energies of the images alongside the original QEq values, using the DFT $+\mathrm{U}$ energy of site A as a convenient zero of energy. This procedure allows a direct comparison of a Minimum Energy Pathway in both models. It 10 is apparent that the activation energy for the pathway is similar in both models $(0.97 \mathrm{eV}$ for the $\mathrm{QEq}$ versus $0.83 \mathrm{eV}$ in $\mathrm{DFT}+\mathrm{U})$. Furthermore, the shape of the pathway energy plots is similar, indicating the $\mathrm{QEq}$ saddle point is geometrically similar to the DFT+U one. For convenience, we also show in ${ }_{15}$ Figure 5 the DFT $+\mathrm{U}$ geometry at some key points on the path and will return to this point in a later section.

Table 2 The excess charge dQ, measured in units of $\mathrm{e}^{-}$, over bulk values for ions labelled in Figs. $4 \mathrm{c}$ and $4 \mathrm{~d}$ due to interstitial insertion.

\begin{tabular}{cccc} 
Fi interstitial & $\begin{array}{l}\text { Hallil QEq } \\
\text { model }\end{array}$ & DFT $+\mathrm{U}$ & \multicolumn{2}{c}{$\begin{array}{l}\text { Fixed oxygen-charge } \\
\text { QEq }\end{array}$} \\
\hline T1 & 0.417 & 0.178 & 0.767 \\
T2 & 0.166 & 0.072 & 0.025 \\
T3 & 0.191 & 0.331 & 0.336 \\
T4 & 0.091 & 0.047 & 0.286 \\
T5 & 0.050 & 0.034 & 0.178 \\
O1 & 0.288 & 0.065 & 0.0 \\
O2 & 0.309 & 0.097 & 0.0 \\
O3 & 0.279 & 0.087 & 0.0 \\
O4 & 0.311 & 0.132 & 0.0 \\
O5 & -0.415 & -0.091 & 0.0
\end{tabular}

Bi interstitial

\begin{tabular}{lccc}
\hline T1 & 0.409 & 0.216 & 0.748 \\
T2 & 0.269 & 0.445 & 0.438 \\
T3 & 0.104 & 0.032 & -0.032 \\
T4 & 0.032 & 0.014 & 0.189 \\
T5 & 0.118 & 0.224 & 0.296 \\
O1 & 0.284 & 0.105 & 0.0 \\
O2 & 0.348 & 0.082 & 0.0 \\
O3 & 0.337 & 0.079 & 0.0 \\
O4 & 0.259 & 0.103 & 0.0 \\
O5 & -0.409 & -0.080 & 0.0 \\
& & & \\
\hline
\end{tabular}

Table 3 Energy barriers (eV) for the forward and backward moves found using the NEB method using the fixed oxygen-charge variant of the $Q E q$ 25 model; the figures in brackets are for the DFT+U estimates (see text).

\begin{tabular}{ccc} 
& Forward & Backward \\
\hline$A-F i$ (exchange) & $0.97(0.83)$ & $1.41(1.40)$ \\
$A-F i$ (direct) & $2.20(---)$ & $2.64(---)$ \\
$B-B i$ (exchange) & $0.47(0.66)$ & $1.94(1.55)$ \\
$B-B i$ (direct) & $0.96(---)$ & $2.43(---)$ \\
$B i-B i i$ (exchange) & $1.31(1.00)$ & $1.35(0.60)$ \\
Bi-Bii (direct) & $2.02(---)$ & $2.06(---)$ \\
$F i-F i i$ (exchange) & $1.31(0.76)$ & $1.53(0.84)$ \\
$A-B$ & $1.12(0.83)$ & $0.14(0.52)$ \\
$A-D$ & $3.49(---)$ & $3.49(---)$ \\
$B-E$ & $1.83(----)$ & $1.83(---)$ \\
$B i-F i$ & $0.81(0.72)$ & $0.77(0.63)$ \\
& & \\
\hline
\end{tabular}

Table 3 shows that an alternative low-energy pathway exists for adatoms to diffuse to interstitial sites, that of $\mathrm{B}-\mathrm{Bi}$ via exchange with an in-plane 5-fold coordinated Ti in the surface 30 trench. In Figure 6 we again compare the MEP found using the NEB method and the modified QEq with that obtained using constrained minimisation in DFT $+U$ along the same pathway, using the starting site B energies in each model as convenient zeros of energy. Again we note the similarity in 35 the shapes of the energy plots along this pathway, lending confidence that the empirical potential provides a pathway that is also feasible in the $\mathrm{DFT}+\mathrm{U}$ methodology. The activation energy is lower in the $\mathrm{QEq}$ scheme at $0.47 \mathrm{eV}$ compared to $0.66 \mathrm{eV}$ for $\mathrm{DFT}+\mathrm{U}$. However, the energy 40 landscape shows that the relative energy of site B over site A is higher in the modified QEq $(0.98 \mathrm{eV})$ than in the DFT+U $(0.31 \mathrm{eV})$. This high energy starting point has the effect of distorting the pathway, lowering the activation energy and moving the saddle point towards the interstitial site Bi. 


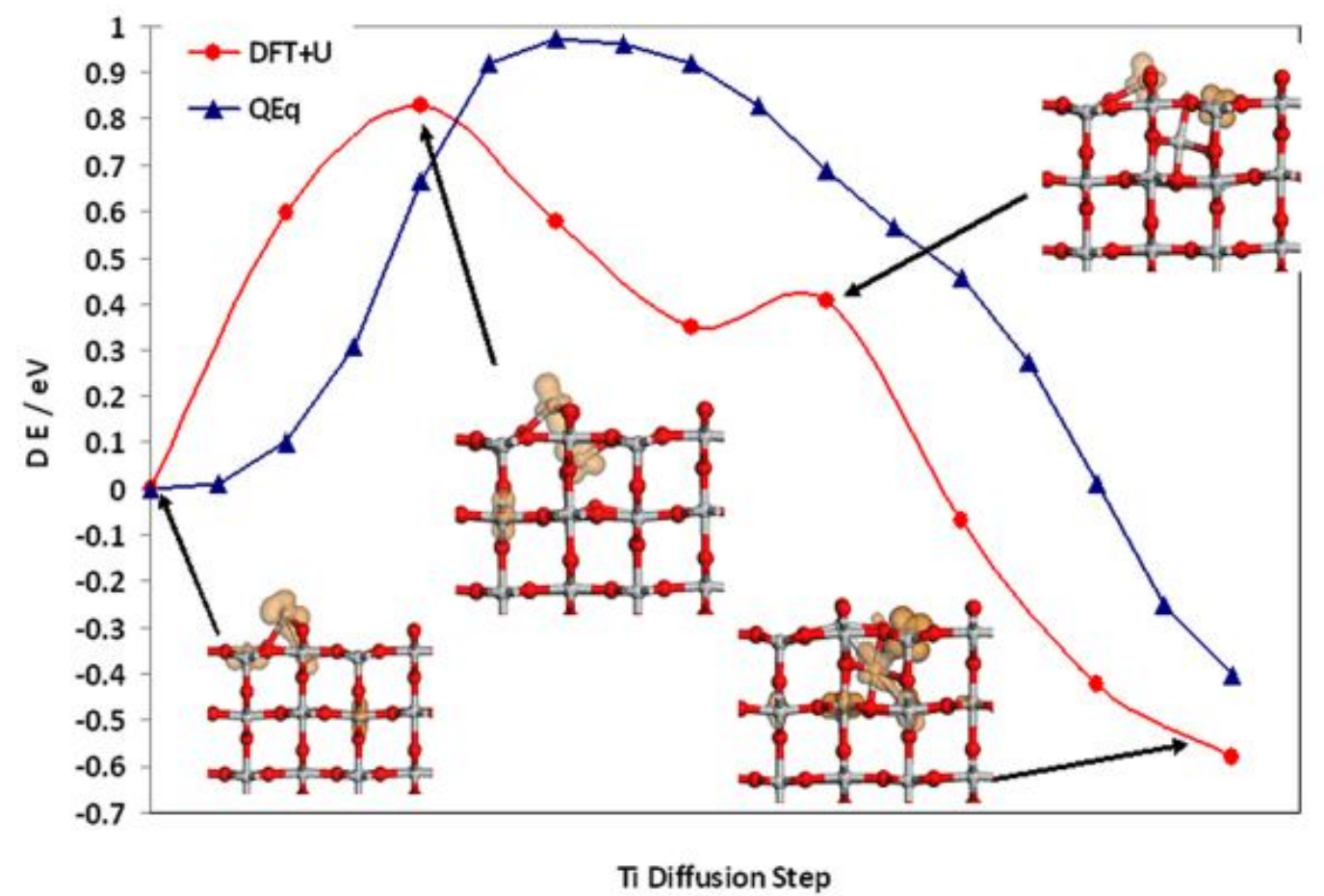

Fig. 5 Minimum Energy Pathways for A-Fi via 6-fold exchange. The structure indicated at selected steps along the MEP includes the DFT+U spin density to show how the charge localisation changes during the migration process.

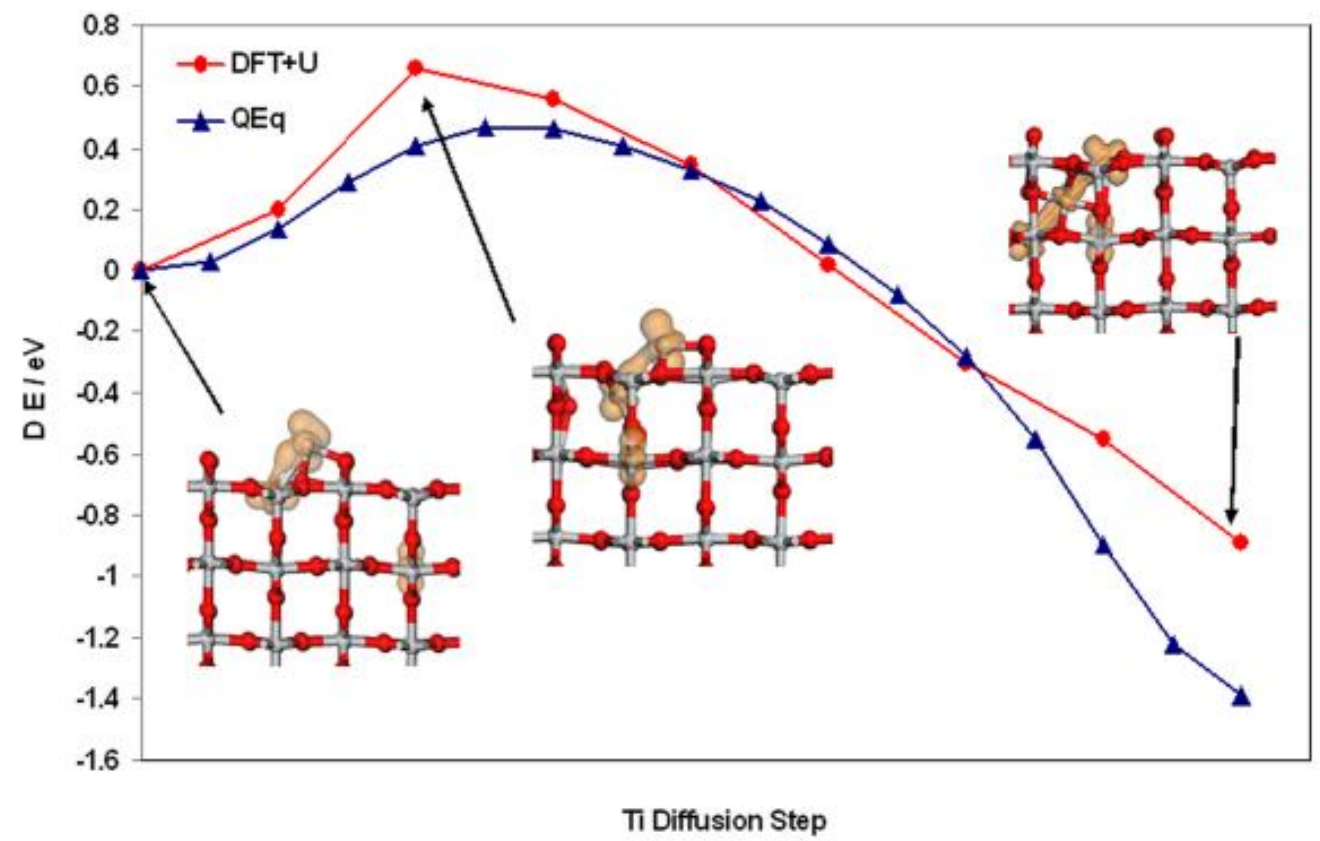

Fig. 6 Minimum Energy Pathways for B-Bi via 5-fold exchange. The structure indicated at selected steps along the MEP includes the DFT+U spin density to show how the charge localisation changes during the migration process 


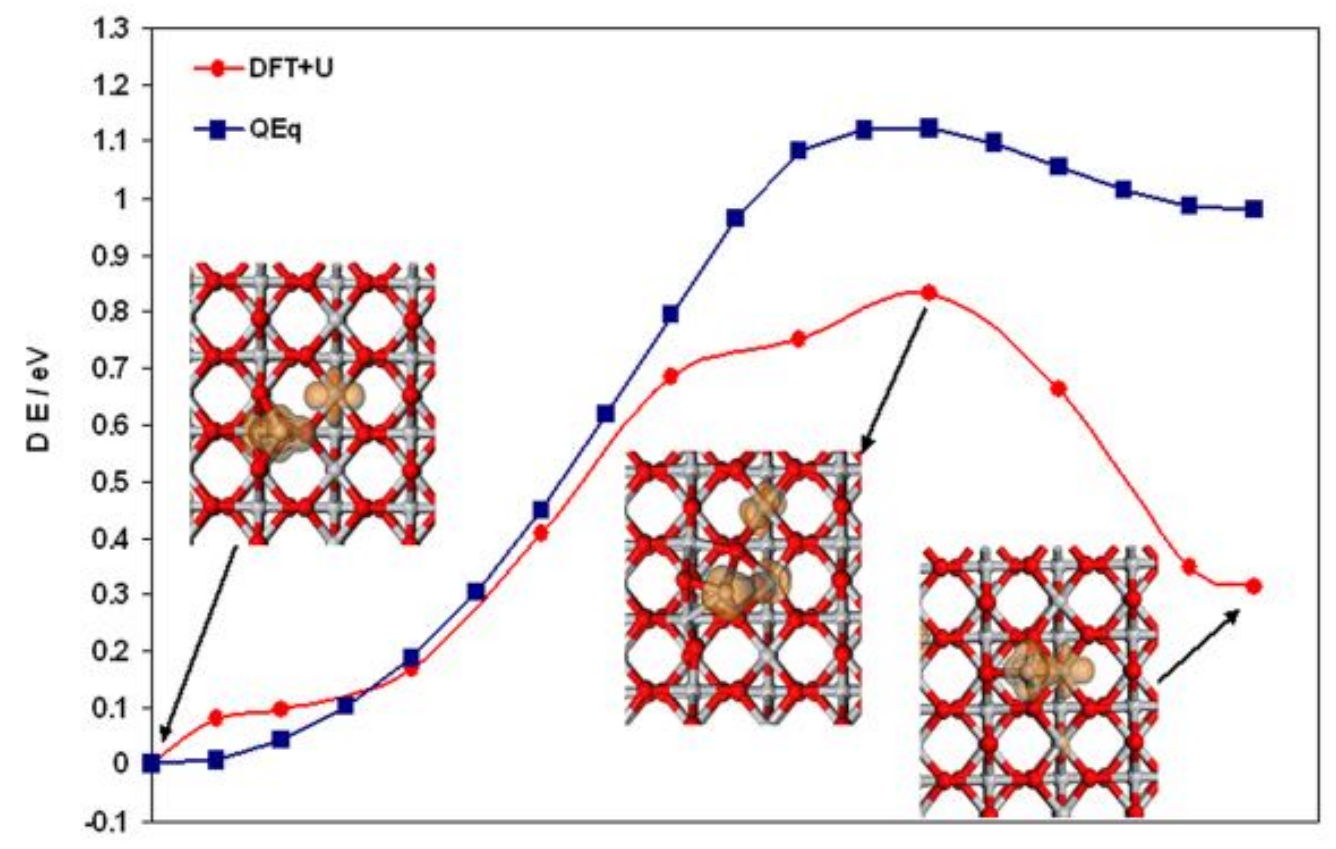

$\pi$ Diffusion Step

Fig. 7 Intra-layer Minimum Energy Pathways from the surface A to surface B site. The structure indicated at selected steps along the MEP includes the $D F T+U$ spin density to show how the charge localisation changes during the migration process

5

While diffusion from a surface B site has a lower activation barrier than from the A site, we need to follow migration of the Ti adatom from the surface A site to the surface B site. To

10 this end, Figure 7, shows the MEP for an adatom diffusing between sites A and B, which has a barrier of $1.12 \mathrm{eV}(\mathrm{QEq}) /$ $0.83 \mathrm{eV}(\mathrm{DFT}+\mathrm{U})$. This emphasises the point that site $\mathrm{B}$ has a rather high energy in the modified QEq model, compared to the DFT+U calculations (see above) and the computed energy 15 barriers also indicate that diffusion from the A to the B site will not be a frequent event. Of particular interest is whether this distorts the activation energy of the adatom to interstitial diffusion. In the modified QEq, the composite pathway A-B$\mathrm{Bi}$ is dominated by the first step with the activation energy $201.12 \mathrm{eV}$, a little higher than the activation energy of $0.97 \mathrm{eV}$ for the aforementioned A-Fi interstitial move. In the DFT $+\mathrm{U}$ model, the composite pathway again is dominated by the A-B activation energy $0.83 \mathrm{eV}$, which is the same activation energy as for the A-Fi interstitial move. Therefore it seems both 25 pathways are competitive in our calculations, although the AB-Bi pathway has two steps, whereas the A-Fi pathway has a single step. The preference appears to be for the A-Fi pathway, although A-B-Bi would be possible on experimental timescales at the relevant growth temperature $>400 \mathrm{~K}^{9,10}$.

${ }_{30}$ To complete our analysis of the interstitial diffusion at the surface, we also study the MEP in the models with subsurface diffusion (data not shown). The intra-layer Bi-Fi move in the first subsurface layer corresponds to the B-A adatom move at the surface. Again the trends in the curves are satisfactorily
35 correlated, showing an almost symmetric shape, with an activation energy of $0.72 \mathrm{eV}$ from $\mathrm{DFT}+\mathrm{U}$, lower than in the surface A-B move. Table 3 shows a summary of the MEP's including diffusion pathways between subsurface layers. The interstitial move Bi-Bii via exchange with a lattice $\mathrm{Ti}$ is found 40 to be favoured over the direct move. The DFT $+\mathrm{U}$ activation energy for this diffusion is $1.00 \mathrm{eV}$, comparing with $1.31 \mathrm{eV}$ for the modified QEq. The Fi-Fii exchange diffusion pathway has a barrier of $0.76 \mathrm{eV}$ in $\mathrm{DFT}+\mathrm{U}$ in good agreement with the value used in ref. 15. The pathways for out diffusion of 45 interstitials torwards the surface from bulk are also given in the backwards pathway column of Table 3 .

\section{Discussion and Conclusions}

The results in this paper present a thorough picture of the ${ }_{50}$ interaction of $\mathrm{Ti}$ atoms with the rutile (110) surface and provide useful insights into the near surface diffusion of interstitial $\mathrm{Ti}$. The Ti adatom is most stable in the surface A site. Although interstitial sites are determined to be more stable, there is a barrier for diffusion from the surface to the ${ }_{55}$ interstitial sites (and vice versa). The most favourable pathway is the surface A site to interstitial Fi site (and vice versa). In this pathway, the Ti adatom moves towards a 6 -fold coordinated lattice $\mathrm{Ti}$ site, which in a concerted motion, moves to the interstitial site. For this pathway, figure 5 shows ${ }_{60}$ the structures of some intermediate steps, clearly showing the concerted motion of both the adatom and the lattice Ti.

In the inserts of figure 5 we show the computed spin density for critical steps. In the A site, the Ti adatom is in the +2 
oxidation state, a surface 5 -fold coordinated $\mathrm{Ti}$ atom is in the +3 oxidation state and the remaining electron of the four introduced by neutral $\mathrm{Ti}$, is spread over a small number of $\mathrm{Ti}$ atoms. In the interstitial $\mathrm{Fi}$, site, the interstitial $\mathrm{Ti}$ and 5 surrounding reduced $\mathrm{Ti}$ atoms have $\mathrm{a}+3$ oxidation state. At the highest point on the MEP, we see that the originally 6-fold coordinated $\mathrm{Ti}$ atom has moved towards the interstitial site and has picked up some charge so that the original adatom now has a +3 oxidation state as does a subsurface Ti atom (the 10 previously reduced 5 -fold surface $\mathrm{Ti}$ atom is now a $\mathrm{Ti}^{4+}$ ion). A second, local, maxima in the MEP arises when the adatom and two surface 5-fold coordinated $\mathrm{Ti}$ atoms have a +3 oxidation state and the interstitial is a $\mathrm{Ti}^{4+}$ ion. By the final stages, one of the surface $\mathrm{Ti}^{3+}$ ions is oxidised to $\mathrm{Ti}^{4+}$ and the 15 interstitial $\mathrm{Ti}$ is now a $\mathrm{Ti}^{3+}$ ion and the system relaxes to a lower energy configuration.

Thus, the Ti diffusion pathway shows a number of changes of $\mathrm{Ti}$ oxidation states for the diffusing atoms and for neighbouring Ti atoms. Our results show that the barrier in the 20 MEP arises at the point at which two (large) $\mathrm{Ti}^{3+}$ ions are rather close to each other, which is an energetically unfavourable situation. Once past this step on the MEP, the reduced $\mathrm{Ti}$ atoms are no longer so close to each other and migration of $\mathrm{Ti}$ proceeds. It is interesting to note that the 25 interstitals donate charge to the 5 fold $\mathrm{Ti}$ in the surface and that these electrons will be available to adsorbates to aid dissociative adsorption ${ }^{13}$. For the re-oxidation of the surface by $\mathrm{O}_{2}$ one may expect these sites to be especially reactive and lead to the creation of $\mathrm{O}$ adatom adsorption close to the $\mathrm{Ti}$ 30 interstitials. These may then facilitate the out-diffusion of $\mathrm{Ti}$ interstitials through the exchange pathways identified here modified by the more stable transition and final states.

For the Ti diffusion pathways in figures 6, 7, we also show the structure and spin densities at important points along the

35 MEP. In all these migration pathways, we observe that the maximum in the barrier is obtained when reduced $\mathrm{Ti}$ species come closest to each other along the migration pathway.

We can compare the diffusion barriers we find with DFT $+\mathrm{U}$ to those found using bare DFT (LDA) in reference 17. In that 40 work, the barrier for the A-Fi interstitialcy move is $1.60 \mathrm{eV}$ (compared to $0.83 \mathrm{eV}$ here) and for the $\mathrm{B}-\mathrm{Bi}$ interstitialcy move it is $1.76 \mathrm{eV}$ (c.f. $0.66 \mathrm{eV}$ ). The contrast for the reverse moves, i.e. the out-diffusion of interstitials to the surface, is even more marked, since the interstitials are highly favoured 45 energetically over adatoms in LDA by $1.91 \mathrm{eV}$ (c.f. $0.53 \mathrm{eV}$ here). These contrasts appear to be due to the role electron localisation plays in stabilising the structures and diffusion pathways, and as stated above, the correct description of this will be important to the understanding of surface dissociative 50 adsorption. There is no doubt that experiments support the lower diffusion barriers found in the DFT $+U$ calculations here. Indeed, some of these diffusion pathways have been (indirectly) measured. In particular a barrier of $0.44 \pm 0.06 \mathrm{eV}$ was found for the in-diffusion of submonolayer of $\mathrm{Ti}$ 55 deposited on near stoichiometric $\mathrm{TiO}_{2}(110)^{11}$. This measurement follows $\mathrm{Ti}^{3+}$ core-level shifted features in photoemission spectroscopy and is thus sensitive to diffusion through several monolayers (the escape depth of photoemitted electrons) but is in reasonable agreement with the lower 60 energy pathways described here. The bulk diffusion has been previously calculated to follow an intersticialcy mechanism with a barrier of $0.225 \mathrm{eV}$ (significantly lower than the barrier of $0.37 \mathrm{eV}$ along the c-axis $)^{43}$.

A comparison of results for the DFT $+\mathrm{U}$ and $\mathrm{QEq}$ descriptions 65 of adsorbed $\mathrm{Ti}$ and $\mathrm{Ti}$ interstitials shows that the modified QEq potential gives results that are in reasonable agreement with the DFT $+\mathrm{U}$ results. The relative stabilities of the various adatom and interstitial sites are in good agreement, as are the charge distributions, as evidenced by plotting the Bader 70 charges for the Ti adatom and interstitial sites. We aim to use the QEq model for studying the interaction of Ti with oxygen and the growth of $\mathrm{TiO}_{2}$ layers, full simulation of which is presently beyond the reach of first principles simulations. The comparison of Ti migration pathways from $\mathrm{DFT}+\mathrm{U}$ and $\mathrm{QEq}$

75 shows that the potential provides a very good description of $\mathrm{Ti}$ migration and will be suitable for dynamic simulation of surface growth processes, in particular where substoichiometric Ti is present. In addition, the QEq potential can also be used for screening of potential structure and 80 diffusion pathways for full simulation of selected structures with first principles approaches.

\section{Acknowledgements}

PAM, CSB and MB acknowledge support from EPSRC grants ${ }_{85}$ EP/C524349 and GR/T18738 and the University of Reading UK. MN acknowledges support from Science Foundation Ireland, "EMOIN" grant number SFI 09/SIRG/I1620 and computing resources provided by the SFI and Higher Education Authority Funded Irish Centre for High End ${ }_{90}$ Computing.

\section{Notes and references}

${ }^{a}$ Department of Chemical and Process Engineering, University of Strathclyde, 75 Montrose Street, Glasgow G1 $1 \mathrm{XJ}$, UK Fax: +44 (0)141 548 2539; Tel: +44 (0)141 548 2385; E-mail: 95 paul.mulheran@strath.ac.uk

${ }^{b}$ Tyndall National Institute, University College Cork, Lee Maltings, Cork, Ireland

Fax: +353-21-4270271; Tel: +353-21-490-4063; E-mail: michael.nolan@tyndall.ie

$100{ }^{c}$ Department of Physics, University of Reading, Whiteknights, Reading, RG6 6AF, UK.

${ }^{d}$ Diamond Light Source Ltd, Diamond House, Harwell Science and Innovation Campus, Didcot OX11 ODE, UK

${ }^{e}$ Department of Mathematical Sciences, Loughborough University, 105 Loughborough LE11 3TU, UK

${ }^{f}$ School of Chemistry, University of Reading, Whiteknights, Reading RG6 6AD, UK. Fax: +44 (0)1189750203; Tel: +44 (0)1183788559; E-mail: r.a.bennett@reading.ac.uk

\section{References}

1. U. Diebold, Surf. Sci. Rep. 2003, 48, 53

2. G.V. Samsonov, The Oxide Handbook, IFI/Plenum Press, New York 1982.

3. L.S. Dubrovinsky, N.A. Dubrovinskaia, V. Swamy, J.Muscat, N.M. 115 Harrison, R. Ahuja, B. Holm, B. Johansson, Nature 2001, 410, 653.

4. R.A. Bennett, S. Poulston, P. Stone and M. Bowker, Phys. Rev. B 1999, 5910341.

5. G.S. Rohrer, V.E. Henrich and D.A. Bonnell, Science 1990, 250, 1239. 
6. G.S. Rohrer, V.E. Henrich and D.A. Bonnell, Surf. Sci. 1992, 278,146.

7. M. Marezio, A. Gauzzi, F. Licci and E. Gilioli, Physica C 2000, 338, 1.

8. H. Onishi and Y. Iwasawa, Phys. Rev. Lett. 1996, 76, 791.

9. P. Stone, R. A. Bennett, and M. Bowker, New Journal of Physics 1999, 1,8 .

10. R. D. Smith, R. A. Bennett, and M. Bowker, Phys. Rev. B 2001, 66, 035409.

11. M. Bowker and R. A. Bennett, J. Phys.: Condens. Matter 2009, 21, 474224; J. Phys.: Condens. Matter 2010, 22, 059801.

10 12. M.A. Henderson, Surf. Sci. 1999, 419, 174.

13. R.A. Bennett, P. Stone and M. Bowker, Faraday. Discuss. 1999, 114, 348 .

14. N. D. McCavish and R. A. Bennett, Topics in Catalysis 2005, 36, 11.

15. S. Wendt P. T. Sprunger, E. Lira, G. K. H. Madsen, Z. Li, J. Ø.

15 Hansen, J. Matthiesen, A. Blekinge-Rasmussen, E. Lægsgaard, B. Hammer, and F. Besenbacher, Science 2008, 320, 1755.

16. R.A. Bennett, P. Stone and M. Bowker, Faraday Discuss. 1999, 114, 267.

17. E. J. Sanville, L. J. Vernon, S. D. Kenny, R. Smith, Y. Moghaddam,

20 C. Browne and P. Mulheran, Phys. Rev. B 2009, 80, 235308.

18. C. M. Yim, C. L. Pang and G. Thornton, Phys. Rev. Lett. 104, 036806, 2010

19. Di Valentin et al. J. Phys. Chem. C 113, 20543, 2009

20. M. Nolan, S.D. Elliott, J.S. Mulley, R.A. Bennett, M. Basham and P.A. Mulheran, Phys. Rev. B 2008, 77, 235424.

21. B. J. Morgan and G. W. Watson, Surf. Sci. 2007, 601, 5034.

22. C. Di Valentin, G. Pacchioni, and A. Selloni, Phys. Rev. Lett. 2006, 97, 16680.

23. A. K. Rappe and W. A. Goddard, J. Phys. Chem 1991, 95, 3358.

30 24. X. W. Zhou, H. N. G. Wadley, J.-S. Filhol, and M. N. Neurock, Phys. Rev. B 2004, 69, 035402.

25. V. Swamy and J. D. Gale, Phys. Rev. B 2000, 62, 5406.

26. V. Swamy, J. Muscat, J. D. Gale, and N. M. Harrison, Surf. Sci. 2002, $504,115$.

35 27. B. S. Thomas, N. A. Marks, and B. D. Begg, Phys. Rev. B 2004, 69, 144122.

28. A. Hallil, R. Tétot, F. Berthier, I. Braems and J. Creuze, Phys. Rev. B 2006, 73, 165406.

29. B. S. Thomas and N. A. Marks, Phys. Rev. B 2007, 76, 167401

40 30. A. Hallil, R. Tétot, F. Berthier, I. Braems and J. Creuze, Phys. Rev. B 2007, 76, 167402 .

31. P.A. Mulheran, C.S. Browne and Y. Moghaddam, Mol. Sim., 2009, 35,532

32. G. Kresse and J. Hafner, Phys. Rev. B, 1994, 49, 14251; G Kresse and

45 J. Furthmüller, Comp. Mat. Sci. 1996, 6, 15

33. P. E. Blöchl, Phys. Rev. B 1994, 50, 17953; D. Joubert and G. Kresse, Phys. Rev. B 1999, 59, 1758.

34. Y. F. Zhang, W. Lin, Y. Li, K. N. Ding, and J. Q. Li, J. Phys. Chem. B, 2005, 109, 19270.

50 35. A. I. Poteryaev, A. I. Lichtenstein, and G. Kotliar, G. Phys. Rev. Lett., 2004, 93, 086401.

36. R. Pentcheva, and W. E. Pickett, Phys. Rev. B, 2006, 74, 035112.

37. D. D. Cuong, B. Lee K. M. Choi H. S. Ahn, S. Han, and J. Lee, Phys. Rev. Lett., 2007, 98, 115503.

55 38. V. I. Anisimov, J. Zaanen, and O. K. Andersen, Phys. Rev B, 1991, 44, 943.

39. S. L. Dudarev, G. A. Botton, S. Y. Savrasov, C. J. Humphreys, and A. P. Sutton, Phys. Rev. B, 1998, 57, 1505.

40. E. Sanville, S. D. Kenny, R. Smith, and G. Henkelman, J. Comp. Chem. 2007, 28, 899.

41. G. Henkelman and H. Johnsson, J. Chem. Phys. 113:9978 (2000)

42. E. Finazzi, C. Di Valentin and G. Pacchioni,J. Phys. Chem. C 113, 3382,2009

43. H. Iddir, S. Öğüt,P. Zapol and N.D.. Browning, Phys. Rev. B, 2007,

$65 \quad 75,073203$ 